\title{
Discrepancies in assessment of patients with rheumatoid arthritis and secondary Sjögren's syndrome by DAS28-ESR and DAS28-CRP
}

\author{
KATARZYNA ROMANOWSKA-PRÓCHNICKA ${ }^{1,2}$, MARZENA OLESIŃSKA ${ }^{l}$, \\ AGNIESZKA PARADOWSKA-GORYCKA ${ }^{3}$, MAŁGORZATA MANCZAK 4 , ANNA FELIS-GIEMZA ${ }^{1}$, \\ PIOTR WOJDASIEWICZ2 ${ }^{2}$ DARIUSZ SZUKIEWICZ ${ }^{2}$
}

${ }^{1}$ Department of General and Experimental Pathology, Warsaw Medical University, Warsaw, Poland

${ }^{2}$ Department and Polyclinic of Systemic Connective Tissue Diseases, National Institute of Geriatrics, Rheumatology and Rehabilitation, Warsaw, Poland

${ }^{3}$ Department of Biochemistry and Molecular Biology, National Institute of Geriatrics, Rheumatology and Rehabilitation, Warsaw, Poland ${ }^{4}$ Department of Epidemiology and Health Promotion, National Institute of Geriatrics, Rheumatology and Rehabilitation, Warsaw, Poland

\begin{abstract}
Objectives: To investigate whether a difference exists between DAS28 from CRP and DAS28 from ESR in patients with rheumatoid arthritis (RA) and secondary Sjögren's syndrome (sSS).

Material and methods: One group comprised patients with RA and SSS, the control group comprised patients with $R A$. The inclusion criteria for the RA and sSS group have been specified as follows: presence of at least one symptom of dryness, and also presence of anti-SS-A and anti-SS-B or at least focus score of one in biopsy.

Results: The disease activity score 28 (DAS28) was assessed using both ESR and CRP in 60 patients with RA and SSS and 59 patients with RA alone. However, concordance between these two methods was good (Cohen's $\kappa$ coefficient $\kappa=0.60$, 95\% CI: 0.45-0.75 in the first group and $\kappa=0.71$, 95\% CI: 0.560.86 in the control group). In the group with RA and $S S S$, the mean value of DAS28-ESR $=5.2$, whereas the mean value of DAS28-CRP $=4.7(p<0.0001)$. In the group with RA alone, mean DAS28-ESR = 4.7 while mean DAS28-CRP = 4.6; no significant difference was identified. Moreover, in RA patients with $\mathrm{sSS}$, mean $E S R=39 \mathrm{~mm} / \mathrm{h}$ compared with mean CRP at $25 \mathrm{mg} / .79 \%$ of all patients demonstrated dysproteinaemia. There were connections between higher ESR and dysproteinaemia. In the control group there was no statistically significant difference between CRP and ESR.

Conclusions: Both DAS28-ESR and DAS28-CRP are useful outcome measures in RA. However, in patients with RA and sSS, DAS28 should be evaluated based on CRP.
\end{abstract}

Key words: secondary Sjögren's syndrome, rheumatoid arthritis, DAS28-CRP, DAS28-ESR.

(Cent Eur J Immunol 2016; 41 (2): 188-194)

\section{Introduction}

Secondary Sjögren's syndrome (sSS) is classified as a connective tissue disease characterized mainly by xerophthalmia and xerostomia, and accompanied by various autoimmune diseases such as rheumatoid arthritis (RA), systemic lupus erythematosus, scleroderma, vasculitis, mixed connective tissue disease, primary biliary cirrhosis or autoimmune thyroiditis [2].

To recognize the sSS presence, one subjective symptom of sickness and positive results of two objective tests for dryness of eyes and mouth are required. The sSS can also be diagnosed without any sicca symptoms, but then the following three criteria should be met: positive results of both objective tests, the presence of antibodies (antiSS-A/Ro and anti-SS-B/La), and inflammatory infiltration in biopsy of minor salivary glands [3].

Prevalence of sSS in RA varies considerably depending on the geographic region and duration of RA. For instance, data from Norway show the lowest prevalence, i.e. $7 \%$, whereas data from Spain reveal a cumulative prevalence of $17 \%$ and $25 \%$ after 10 and 30 years of the disease, respectively [4]. According to Andonopoulos et al., 5\% of RA patients had clinical manifestations of sSS and $20 \%$ demonstrated sub-clinical sSS [5].

Patients with secondary SS and RA require special attention regarding evaluation of specific systemic com-

Correspondence: Katarzyna Romanowska-Próchnicka, PhD, MD, Department of General and Experimental Pathology, Warsaw Medical University, Pawińskiego 3 C, 02-106 Warsaw, Poland, tel. +48 2257 20 710, fax +48 22646 78 94, e-mail: katarzyna.prochnicka@ gmail.com Submitted: 5.09.2015, Accepted: 26.04.2016 
plications like inflammatory pulmonary disease or polyneuropathy.

DAS28 is an important outcome measure of RA activity. In a number of European countries, DAS28 is conclusive in determining intensity of treatment.

The aim of this study is to evaluate whether the difference in the values of the DAS28 calculated taking into account diverse parameters of inflammatory activity (ESR or CRP) is clinically relevant. In case the discrepancy is relevant, the study should indicate which parameter is more valuable for assessing activity of RA with sSS.

\section{Material and methods}

\section{Patients}

To capture data regarding the study we queried our medical records database of the Connective Tissue Disease Department in the Institute of Rheumatology in Warsaw from 2009 through 2011 seeking to identify all patients with RA and sSS. Initially, 1980 patients were identified with RA who had been diagnosed according to the American College of Rheumatology criteria laid down in 1987. We have separated 119 patients with RA. After a review of medical records, only patients with RA and sSS who had been diagnosed based on the 2002 revised version of the American-European Consensus Group criteria were selected. Eventually, only 60 subjects registered with the ICD-10 code for sSS and RA (M35.0 and M05) were included in the research group. 59 patients were in the control group with RA only.

\section{Clinical and laboratory assessment}

The data were extracted from medical records retrospectively based on their fulfilment of inclusion criteria for our study. The inclusion criteria have been specified as follows:

- radiological confirmation of RA (criteria for RA),

- presence of data on DAS28-CRP and DAS28-ESR,

- presence of at least one subjective symptom of dry eyes or dry mouth (criteria for SS),

- one objective symptom of dryness,

- presence of specific antibodies SS-A and SS-B,

- in biopsy at least focus score of one.

Subjective ocular symptoms were usually described in the medical records as present or absent, and included symptoms of dry eyes lasting over 3 months, foreign object sensation in the eyes, or subjective oral symptoms such as symptoms of dry mouth lasting over 3 months or swollen salivary glands. The objective ocular test was performed using Schirmer's test. Schirmer's test without anaesthesia consists in corneal staining assessment during over 5 minutes and is performed with eyes closed by inserting a Schirmer filter paper strip into the eye and reading the result after 5 minutes. The Schirmer test result is abnormal when during 5 minutes the tears flow is less than $5 \mathrm{~mm}$.

Furthermore, (SSA)/Ro and/or (SSB)/La was collected. In addition, histopathological examination of lymphocytic infiltration at salivary glands with at least focus score of one was performed.

The evaluated medical records contained basic clinical laboratory tests and included rheumatoid factor (RF), anticyclic citrullinated peptide (CCP) antibodies, as well as morphology, inflammatory markers (ESR and CRP), and proteinogram. Only patients with both DAS28-ESR and DAS28-CRP data available at baseline had been selected. DAS 28 embraces swollen joint counts (SJCs) and tender joint counts (TJCs), a self-determined assessment of patient's general health on visual analogue scale (VAS) from 0 to 10, and acute-phase response factors ESR or CRP. According to EULAR recommendations, DAS28 scores represent the following disease activities: remission $(<2.6)$, mild $(\geq 2.6,<3.2)$, moderate $(\geq 3.2,<5.1)$ or severe $(\geq 5.1)$. DAS28 values were calculated using the following equations:

DAS28-CRP $=0.56_{-}{ }^{\vee}(\mathrm{TJC}-28)+0.28_{-}{ }^{\vee}(\mathrm{SJC}-28)+$ $0.014 \_\mathrm{GH}+0.36 \_\mathrm{ln}(\mathrm{CRP}+1)+0.96$,

DAS28-ESR $=0.56_{-}$( $(\mathrm{TJC}-28)+0.28 \_$( $(\mathrm{SJC}-28)+$ 0.014_GH+0.70_ln (ESR).

\section{Statistical analysis}

The following methods were used for statistical analysis: STATISTICA release 10 from Stat Soft Poland, SAS System 10.0 SAS Institute Inc. Institute of Rheumatology, Warsaw, Poland. Normal distribution data have been presented as mean and standard deviations, whereas abnormal distribution data as median and interquartile range. Depending on their distribution, data were analyzed using Student $t$-test or Mann-Whitney $U$ test. $P$-values $<0.05$ were considered statistically significant. Cohen's $\kappa$ coefficient with linear weighting was used to measure agreement between DAS28-ESR and DAS28-CRP values. In order to assess the variation between DAS28-ESR and DAS28CRP, the Bland-Altman plot was used.

\section{Results}

119 out of 1980 patients registered in the Institute of Rheumatology database who suffer from RA have fulfilled inclusion criteria for the research study. One group comprised 60 patients with RA and sSS, second - the control group - patients with RA alone. In the patients with RA and sSS group, mean age was 57 (range: 33-81) years. There were 56 female participants (93\%) and only 4 male $(7 \%)$. Baseline characteristics of the patients are presented in Table 1. The control group comprised 59 patients with RA. Mean age of the control group patients was 51.6 
Table 1. Baseline characteristics of the patients with RA and sSS and the control group

\begin{tabular}{lccc}
\hline Parameter & RA patients with sSS & RA patients & $\begin{array}{c}\boldsymbol{p} \text {-Mann-Whitney test } \\
\boldsymbol{p}^{*} \text {-Student's } \boldsymbol{t} \text {-test }\end{array}$ \\
\hline Age [yr], mean (SD) & $57(11)$ & $51.6(13.7)$ & $0.027^{*}$ \\
\hline ESR [mm/h], median & 34.5 & 15 & $<0.001$ \\
\hline CRP [mg/dl], median & 14 & 16 & 0.43 \\
\hline VAS [mm], median & 51 & 60 & 0.83 \\
\hline Tender joints, median & 8 & 8 & 0.52 \\
\hline Swollen joints, median & 4 & 3 & 0.75 \\
\hline DAS-28 from ESR, mean (SD) & $5.2(1.3)$ & $4.7(1.5)$ & $0.10^{*}$ \\
\hline DAS-28 from CRP, mean (SD) & $4.7(1.2)$ & $4.6(1.4)$ & $0.84^{*}$
\end{tabular}

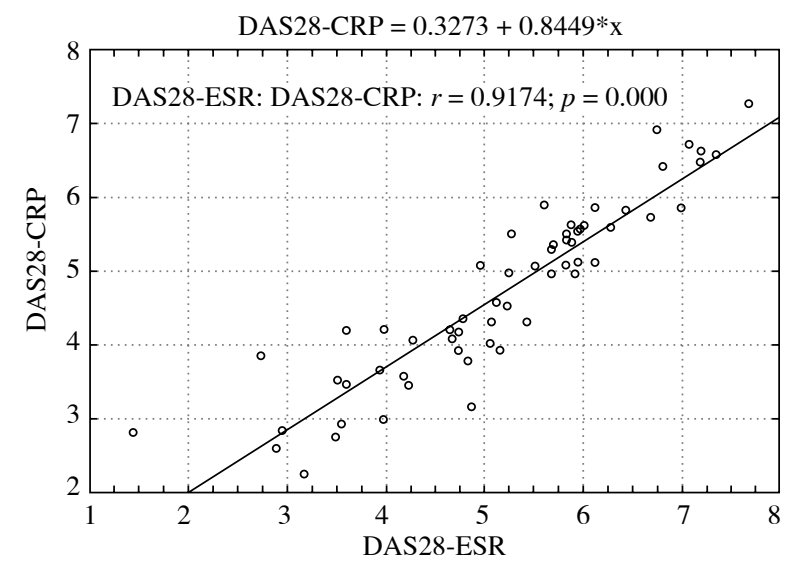

Fig. 1. Scatter Plot of DAS28-CRP ( $y$ axis) vs. DAS28ESR ( $x$ axis) values with a regression line in the group patients with RA and sSS. Each point corresponds to a single patient's data

(range: 23-77) years. There were also only 4 males and 55 females.

In the group of patients with RA and sSS, 50 out of 60 patients $(83 \%)$ have complained of dry eyes, of whom $15(25 \%)$ have complained of dry mouth also. Moreover, 30 out of 52 patients $(87 \%)$ have obtained a positive result of Schirmer test, i.e. less than $<5$ mm, 17 (33\%) demonstrated border tears flow, while only $9 \%$ normal tears flow. With regard to 8 patients, Schirmer's test data were unavailable. With reference to 52 out of 60 patients (87\%), data of collected ANA antibodies indicated that 28 of them (54\%) show positive ANA antibodies, 13 (26\%) SSA/Ro antibodies, and $6(12 \%) \mathrm{SSB} / \mathrm{La}$ antibodies. The salivary gland biopsy has been performed in 50 of our patients (83\%), whereas 47 out of 50 (94\%) obtained focus score of one and more than one.

A group of 46 patients with RA and sSS (82\%) demonstrated a positive RF, however, for the remaining 4 patients

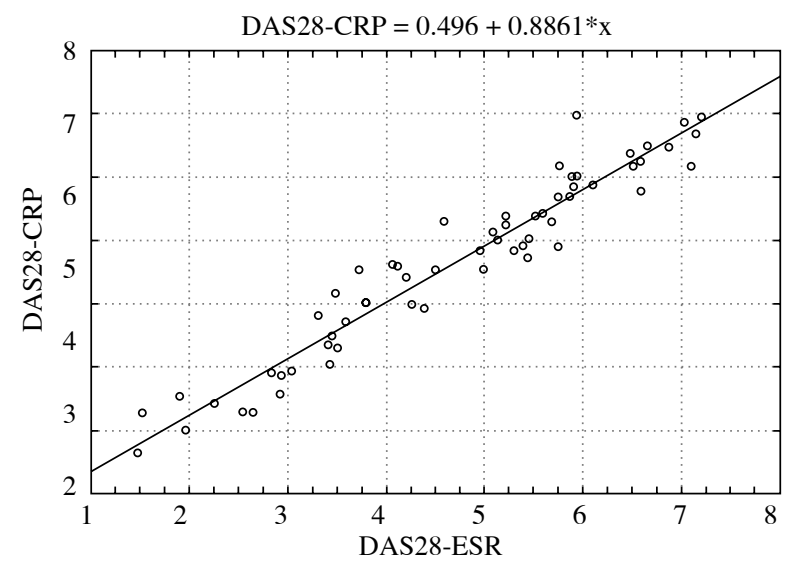

Fig. 2. Scatter Plot of DAS28-CRP ( $y$ axis) vs. DAS28ESR ( $x$ axis) values with a reggression line in the group patients with RA. Each point corresponds to a single patient's data

there were no data. Out of 31 patients $(52 \%)$ with available data of anti-CCP antibodies, 28 (90\%) presented positive anti-CCP antibodies. Divergently a group of RA patients from the control group reported a positive RF in $51 \%$ and positive antiCCP antibodies in $78 \%$.

It should be noted that in patients with RA and sSS, the mean value of ESR was $39 \mathrm{~mm} / \mathrm{hr}$, whereas of CRP, $25 \mathrm{mg} / \mathrm{l}$. On the contrary in RA patients the mean value of ESR was $15 \mathrm{~mm} / \mathrm{hr}$, and almost similar CRP - $16 \mathrm{mg} / \mathrm{l}$.

Linear regression analysis revealed a significant correlation between DAS28-ESR and DAS28-CRP - Pearson correlation coefficients $r=0.9, p<0.0001$ (Fig. 1) in the first group of patients with RA and sSS. In the control group, there was the same correlation between DAS28ESR and DAS28-CRP - Pearson correlation coefficients $r=0.91, p<0.02$ (Fig. 2). Moreover, agreement between DAS28-ESR and DAS28-CRP was good; Cohen's kappa coefficient with linear weighting $\kappa=0.60$; 95\% CI: 0.45 - 
Bland-Altman graph comparing the DAS28-ESR and DAS28-CRP

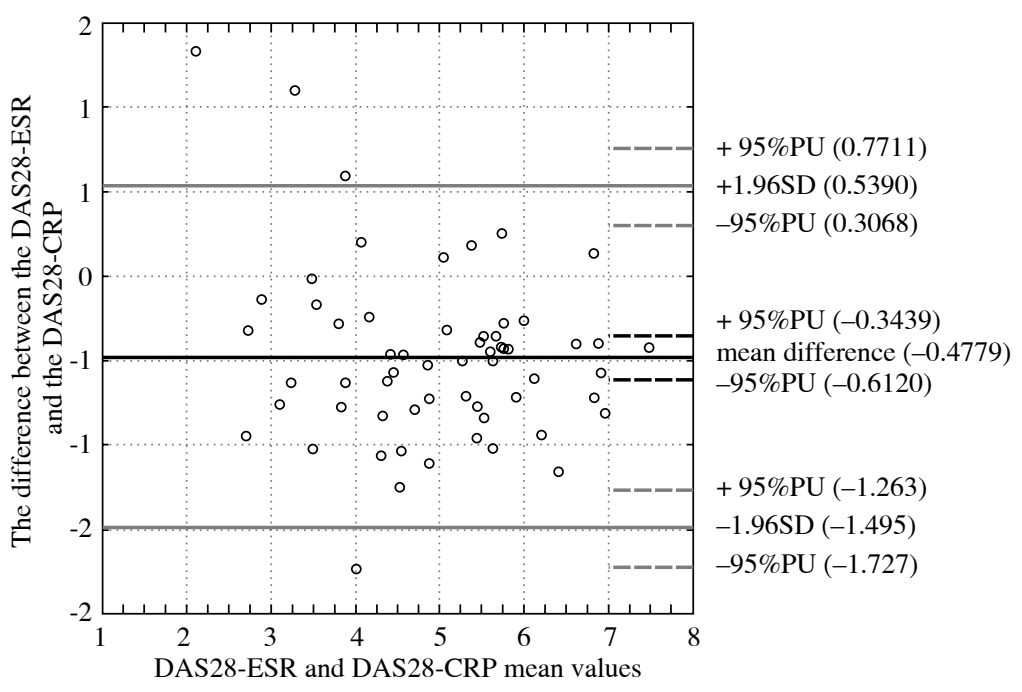

Fig. 3. Bland-Altman plot analysis of DAS28 absolute values. The ordinate scale represents the difference between DAS28-CRP and DAS28-ESR (DAS28-CRP minus DAS28-ESR). The abscissa scale represents the average DAS28ESR and DAS28-CRP (DAS28-ESR plus DAS28-CRP divided by 2). The middle dashed line indicates the bias (mean difference), and the upper and lower dotted lines represent the limits of agreement (mean $\pm 2 \mathrm{SD}$ ). The solid red line represents the regression line of the average difference

0.75 in the RA and sSS group, and Bland-Altman analysis (Fig. 3) also shows good agreement between both methods. Despite this concordance, we found that in patients with RA and sSS, DAS28 calculated from ESR was significantly higher than calculated from CRP. Mean DAS28-ESR = 5.2, while mean DAS28-CRP $=4.7$. A relevant difference was identified with $p<0.0001$. On the other hand, in the control group (patients with RA), mean DAS28-ESR=4.7 while mean DAS28-CRP $=4.6$; no significant difference was identified with $p>0.05(p=0.91)$.

The patients were divided into 4 groups: with remission, low, medium and high disease activity score (see Table 2). In our study, in RA patients with sSS, DAS28ESR $\geq 5.1$ has been reported in 33 patients $(55 \%)$, while DAS28-CRP $\geq 5.1$ in 23 patients (38\%). Congruence between values obtained from ESR and CRP is shown in Table 2. Concordant values are presented in the diagonal of the table. Full agreement has been found for DAS28 values calculated from CRP; for every DAS28-CRP $\geq 5.1$, the DAS28-ESR is also $\geq 5$.1. On the other hand, 10 patients for whom DAS28-CRP ranged from 3.2 to 5.1 had the DAS28-ESR value over 5.1. As a result, the biggest discrepancies were found for high DAS28 values, especially in the range from 3.2 to 5.1, and over 5.1. Compared to the control group, there are no large differences in the value of DAS28-CRP and DAS28-ESR between 2.6 and 5.1. DAS28-ESR $\geq 5.1$ was measured in 25 patients $(42.7 \%)$, while DAS28-CRP $\geq 5.1$ in 29 patients (49\%).
The objective of the study was to find a relationship between higher ESR and hypergammaglobulinemia in patients with RA and sSS. No statistically significant results were obtained in the case of higher ESR and hypergammaglobulinemia, however, connections were found between higher ESR and dysproteinaemia. Patients with RA and sSS who presented dysproteinaemia obtained median ESR over $42 \mathrm{~mm} / \mathrm{h}$, whereas participants without dysproteinaemia had lower median ESR, only $22 \mathrm{~mm} / \mathrm{h}(p<0.011)$ (Table 3).

\section{Discussion}

This study evaluating 60 patients with RA with sSS revealed that values of DAS28-CRP are significantly lower than those of DAS28-ESR. Discrepancies were found in patients with moderate disease activity (DAS28 from 3.2 to 5.1) (DAS28-CRP - 30 patients, DAS28-ESR - 23 patients) and a high disease activity score (DAS28 above 5.1) (DAS28-CRP - 23 patients, DAS28-ESR - 33 patients). These observations have implications for clinical evaluations since a high disease activity score qualifies patients for biological treatment. The afore-mentioned findings change the therapeutic approach, hence, this study is aimed to prove that patients with RA and sSS have different outcomes in assessment from DAS28-ESR than from DAS28-CRP and, therefore, evaluations from DAS28$\mathrm{CRP}$ are recommended. 
Table 2. Concordance between DAS28 values using CRP and ESR in RA patients with SS and the control group

\begin{tabular}{|c|c|c|c|c|c|c|}
\hline \multirow{2}{*}{\multicolumn{2}{|c|}{$\begin{array}{l}\text { DAS in RA patients } \\
\text { with sSS }\end{array}$}} & \multicolumn{4}{|c|}{ CRP } & \multirow{3}{*}{$\begin{array}{c}\text { Total } \\
1\end{array}$} \\
\hline & & \multirow{2}{*}{$\begin{array}{c}<\mathbf{2 . 6} \\
0\end{array}$} & \multirow{2}{*}{$\begin{array}{c}\text { from } 2.6 \text { to } 3.1 \\
1 \\
\end{array}$} & \multirow{2}{*}{$\begin{array}{c}\text { from } 3.2 \text { to } 5.1 \\
0 \\
\end{array}$} & \multirow{2}{*}{$\begin{array}{c}\geq \mathbf{5 . 1} \\
0\end{array}$} & \\
\hline ESR & $<2.6$ & & & & & \\
\hline & from 2.6 to 3.1 & 0 & 2 & 1 & 0 & 3 \\
\hline & from 3.2 to 5.1 & 1 & 3 & 19 & 0 & 23 \\
\hline & $\geq 5.1$ & 0 & 0 & 10 & 23 & 33 \\
\hline \multicolumn{2}{|r|}{ Total } & 1 & 6 & 30 & 23 & 60 \\
\hline \multirow{2}{*}{\multicolumn{2}{|c|}{$\begin{array}{c}\text { DAS } \\
\text { In RA patients }\end{array}$}} & \multicolumn{4}{|c|}{ CRP } & Total \\
\hline & & $<2.6$ & from 2.6 to 3.1 & from 3.2 to 5.1 & $\geq 5.1$ & \\
\hline \multirow[t]{5}{*}{ ESR } & $<2.6$ & 6 & 2 & 0 & 0 & 8 \\
\hline & from 2.6 to 3.1 & 0 & 3 & 1 & 0 & 4 \\
\hline & from 3.2 to 5.1 & 0 & 0 & 16 & 6 & 22 \\
\hline & $\geq 5.1$ & 0 & 0 & 2 & 23 & 25 \\
\hline & Total & 6 & 5 & 19 & 29 & 59 \\
\hline
\end{tabular}

Table 3. Differences in ESR and CRP values in groups of patients with RA and sSS, dysproteinaemia and hypergammaglobulinemia; all values are median and IQR - interquartile range

\begin{tabular}{|c|c|c|c|}
\hline & $\begin{array}{l}\text { Dysproteinaemia - present } \\
\qquad n=41\end{array}$ & $\begin{array}{l}\text { Dysproteinaemia - absent } \\
\qquad n=11\end{array}$ & $\begin{array}{c}p \\
\text { test } U\end{array}$ \\
\hline ESR & $42(26-56)$ & $22(16-36)$ & 0.011 \\
\hline \multirow[t]{2}{*}{ CRP } & $16(8-31)$ & $11(6-19)$ & 0.272 \\
\hline & $\begin{array}{c}\text { Hypergammaglobulinemia - present } \\
\qquad n=24\end{array}$ & $\begin{array}{c}\text { Hypergammaglobulinemia - absent } \\
\qquad n=28\end{array}$ & \\
\hline ESR & 43.5 (25-58.5) & 33.5 (19.5-49) & 0.244 \\
\hline CRP & $11.5(5.5-30.5)$ & $16.5(9.5-24.5)$ & 0.666 \\
\hline
\end{tabular}

In several previous studies [6-9], DAS28 from CRP and DAS28 from ESR were compared. Fransen et al. [9] reported that not only the DAS28-CRP 4.38 [SD 1.47] and DAS 28 ESR 4.41 [SD 1.27] are similar, but also that CRP could be replaced by ESR. According to Wells et al. [6], most patients were classified as having the same EULAR state regardless of which DAS28 definition has been employed. Moreover, Inoue et al. [8] have shown that DAS28-CRP can be used as an alternative to the DAS28-ESR given the strong linear relationship between DAS28-ESR and DAS28-CRP values (correlation coefficient 0.946) [8]. By contrast, in our research group, the said correlation coefficient is equal to only 0.73 . In comparison to his predecessors, Matsui et al. [7] demonstrated that DAS28-CRP significantly underestimated disease activity and overestimated the improvement in disease activity compared with DAS28-ESR. Our study has revealed that the mean value of DAS28-CRP (4.71, SD 1.2) was significantly smaller than the mean value of DAS28-ESR (5.27, SD 1.32) $(p<0.0001)$ in a group with RA and sSS (Table 1). There were no significant statistical correlations in the control group with RA. Nevertheless, the changes of DAS28 values in DAS28-ESR and DAS28-CRP in both groups were very similar and closely correlated. Although the values of DAS28-ESR and those of DAS28-CRP were comparable in the reports of Wells et al. [6] and Fransen et al. [9], our study did not show similar results (Table 2).

In contrast with the previous research, this study covers a different group of patients than the statistical rheumatoid patients. In our research, not only patients with RA have undergone examination, but also those with sSS. That syndrome is responsible for changes in immunoglobulin, especially activation of polyclonal B-cell leading to chronic hypergammaglobulinemia and increased levels 
of $\beta 2$-microglobulinemia [10] which affect the ESR and cause higher ESR. Apart from the aforementioned, ESR can also be affected by such factors as sex, age, immunoglobulin levels, and abnormal size or shape of red blood cells. In chronic diseases, the ESR-elevation is considered to be caused by fibrinogen, mono- or polyclonal increase in $\operatorname{IgG}, \operatorname{Ig} \mathrm{A}$, and $\operatorname{IgM}$, alone or in combinations [11]. Normal values of ESR for females are higher than for males and increase with age. Due to the fact that the study group was dominated by women (93\%), that factor could possibly have influenced the results.

In an inflammatory process, the high proportion of fibrinogen in the blood causes red blood cells to stick to each other. The red cells form stacks called rouleaux which settle faster in ESR. Fibrinogen is considered to be responsible for the rouleaux phenomenon, however, it has a co-influence on immunoglobulin to induce the rouleaux type of aggregates and high ESR. High ESR is a result of either rouleaux type aggregates where fibrinogen is dominant, or immunologic type aggregates where $\operatorname{IgG}, \operatorname{IgA}$ or IgM are the dominant proteins [11]. On the other hand, total leukocyte and absolute neutrophil counts are not correlated with either ESR or haematocrit standardised ESR. A strong correlation $(r=0.75)$ was found between fibrinogen levels and haematocrit standardised ESR [14]. In our study this correlation has not been measured. However, in our study polyclonal gammopathy and dysproteinaemia were reported. Serum protein electrophoresis is a laboratory examination that is commonly used to identify two major types of proteins: albumin and globulins. The five components (globulins) are labeled $\alpha_{1}, \alpha_{2}, \beta_{1}, \beta_{2}$, and $\gamma$. Dysproteinaemia in our study means an abnormality of the protein content of the blood, involving the immunoglobulins ( $\alpha, \beta$ and $\gamma$ globulins). Polyclonal gammopathy can be observed in patients with SS. Polyclonal hypergammaglobulinemia and elevated $\alpha_{2}$-globulins are often seen. In our study $46 \%$ of patients demonstrated hypergammaglobulinemia and $69 \%$ dysproteinaemia, hence, higher ESR can be determined by this pathology. A retrospective cohort study of 148 patients from the Mayo Clinic showed that in patients with RA, $\gamma$ globulin levels are elevated in $37 \%$ of patients, and are reported to correlate with disease activity [16]. Our study also revealed that patients with dysproteinaemia have higher ESR, what proved to be a significant correlated factor. Furthermore, ESR tends to show the disease activity over the course of a few weeks, whereas CRP can develop more short-term changes in the disease activity. Moreover, CRP is less dependable on sex, age, and fibrinogen and immunoglobulin levels [15]. Thus, CRP tends to be a better factor than ESR.

\section{Conclusions}

In conclusion, both DAS28-ESR and DAS28-CRP are useful outcome measures in RA. However, our study pro- vides evidence that in patients with RA and sSS, DAS28 should be evaluated based on CRP.

\section{Key messages}

Both DAS28-ESR and DAS28-CRP are useful outcome measures in RA. Although our results are based on small numbers, they confirm that in patients with RA and sSS, DAS28 should be evaluated from CRP.

\section{The authors declare no conflict of interest.}

\section{References}

1. Jonsson R, Haga HJ, Gordon T (2001): Sjogren's syndrome. In: Koopeman WJ ed. Arthritis and Allied Conditions.A Textbook of Rheumalology. $14^{\text {th }}$ ed. Lippincott \& Williams \& Wilkins, Philadelphia 1736-1759.

2. Coll J, Rives A, Grino MC, et al. (1987): Prevalence of Sjogren's syndrome in autoimmune diseases. Ann Rheum Dis 46: 286-289.

3. Seror R, Ravaud P, Bowman SJ, et al. (2010): EULAR Sjogren's syndrome disease activity index:development of a consensus systemic disease activity index for primary Sjogren's syndrome. Ann Rheum Dis 69: 1103-1109.

4. Haga HJ, Naderi Y, Moreno AM, Peen E (2012): A study of the prevalence of sicca symptomsand secondary Sjögren's syndrome in patients with rheumatoid arthritis, andits association to disease activity and treatment profile. Int J Rheum Dis 15: 284-288.

5. Andonopoulos AP, Drosos AA, Skopouli FN, et al. (1987): Secondary Sjogren's syndrome in rheumatoid arthritis. J Rheumatol 14: 1098-1103.

6. Wells G, Becker JC, Teng J, et al. (2009): Validation of the 28-joint Disease Activity Score (DAS28) and European League Against Rheumatism responsecriteria based on C-reactive protein against disease progression in patients with rheumatoid arthritis, and comparison with the DAS28 based on erythrocyte sedimentation rate. Ann Rheum Dis 68: 954960.

7. Matsui T, Kuga Y, Kaneko A, et al. (2007): Disease Activity Score DAS28) using CRP underestimates the disease activity and overestimates the EULAR response criteria compared with DAS28 using ESR in a large observational cohort of rheumatoid arthritis patients in Japan. Ann Rheum Dis 66: 1221-1226.

8. Inoue E, Yamanaka H, Hara M, et al. (2007): Comparison of DiseaseActivity Score (DAS)28-erythrocyte sedimentation rate and DAS28-C-reactive proteinthreshold values. Ann Rheum Dis 66: 407-409.

9. Fransen J, Welsing PM, de Keijzer RM, van Riel PL (2004): Disease activity scores using C-reactive protein: CRP may replace ESR in the assessment of RA disease activity[abstract]. Ann Rheum Dis 62 (Suppl 1): 151.

10. Fox RI, Stern M, Michelson P (2000): Update in Sjögren syndrome. Curr Opin Rheumatol 12: 391-398.

11. Talstad I, Scheie P, Dalen H, Roli J (1983): Influence of plasma proteins on erythrocyte morphology and sedimentation. Scand J Haematol 31: 478-484. 
12. Talstad I, Haugen HF (1979): The relationship between the erythrocyte sedimentation rate (ESR) and plasma proteins in clinical materials and models. Scand J Clin Lab Invest 39: 519-524.

13. Reinhart WH (1988): Blood sedimentation - a simple and useful test? Schweiz Med Wochenschr 118: 839-844.

14. Allen BV (1988): Relationships between the erythrocyte sedimentation rate, plasma proteins and viscosity, and leucocyte counts in thoroughbred racehorses. Vet Rec 122: 329-332.

15. Kushner I (1991): C-reactive protein in rheumatology. Arthritis Rheum 34: 1065-1068.

16. Dispenzieri A, Gertz MA, Therneau TM, Kyle RA "Polyclonal Gammopathy - A Retrospective Cohort Study of 148 Patients" http://www.2ndchance.info/ScienceBasedVaccinationSchedule-MayoClinic2002.pdf Volume 27, number 3. 